\title{
Chancen des inklusionsorientierten Sachunterrichts für didaktisch-diagnostisches Handeln - Konzeptionelle und methodologisch-methodische Grundlagen eines forschungsbasierten Entwicklungsansatzes für die Lehrer*innenbildung
}

\author{
René Schroeder, Eva Blumberg, Brigitte Kottmann, Susanne Miller \& Anne Reh
}

\begin{abstract}
Zusammenfassung
In der Herausforderung Unterricht inklusionsbezogen weiterzuentwickeln, manifestiert sich unter anderem die Forderung nach der Qualifizierung von (angehenden) Lehrkräften bzgl. der Anwendung und Umsetzung einer alltagsintegrierten Diagnostik mit dem Ziel, Kinder individualisiert und gleichzeitig mit Bezug auf einen gemeinsamen Lerngegenstand zu unterrichten. Dabei ist ein didaktisch-diagnostisches Handeln aller Lehrkräfte gefordert, welches im Kontext einer professionellen und inklusionsorientierten Gestaltung sowie einer angemessenen Begleitung und Förderung von Lern- und Entwicklungsprozessen steht. In der Konzeption entsprechender Aus- und Weiterbildungsformate setzt das geplante Projekt DiPoSa (Didaktisch-diagnostische Potentiale des inklusionsorientierten Sachunterrichts) an. Ziel ist es durch einen DesignBased-Research-Ansatz (DBR) Videovignetten zu erstellen und zu evaluieren, mittels derer entsprechende Kompetenzen von (angehenden) Lehrkräften im Sachunterricht weiterentwickelt werden können. Dabei bietet insbesondere der Sachunterricht als vielperspektivisches Integrationsfach der Grundschule einen passenden Rahmen, kindliche Lernpotentiale als Ausgangspunkt für didaktisch-diagnostisches Handeln nutzbar zu machen. Denn in aktuellen inklusionsorientierten Ansätzen des Sachunterrichts sind sowohl reformpädagogisch orientierte als auch offene Konzepte und Modelle des adaptiven Unterrichts zu finden, die sich an den unterschiedlichen Programmatiken der Umsetzung von individueller Förderung orientieren. Im folgenden Beitrag werden die dem geplanten Projekt zugrundeliegenden theoretischen und empirischen Zugänge erläutert und die forschungsmethodische Umsetzung durch einen DBR-Ansatz als methodologische Basis für eine Theorie-Praxis-verzahnende Forschungsperspektive auf diagnostisches Handeln sowie die Förderung diagnostischer Handlungskompetenzen durch Videovignetten im Kontext der Aus- und Weiterbildung von (Sachunterrichts-)Lehrkräften diskutiert.
\end{abstract}

\section{Schlagworte}

Didaktische Diagnostik, inklusionsorientierter Sachunterricht; Lehrkräfteprofessionalisierung; Videovignetten; Design-Based-Research

\section{Title}

Potentials of formative assessment practices in social studies and science in inclusive primary education - conceptual and methodological implications of a design-based-research-based approach for teacher education

\section{Abstract}

The challenge to further promote practices of inclusive teaching, requires the development of assessment literacy. Combined with the aim to teach all children in a collaborative learning environment, qualification of teachers (in training) in the application and implementation of 
formative assessment as an integrated daily routine is needed. Therefore, the project DiPoSa focuses on the development of appropriate training and qualification formats. The project heads to create and evaluate video vignettes using a design-based-research approach (DBR), which can then be used to further develop assessment literacy of teachers in social studies and science in primary education. As a multi-perspective integrative subject in elementary school social studies and science in primary education offers a suitable framework for practices of formative assessment taking children's learning potentials as a serious concern. Foundations can be seen in current inclusive approaches to social studies and science in primary education, where reform pedagogically orientation and open concepts can as well be found as models of adaptive teaching. Although there are different theoretical and programmatic implementations, both perspectives share an orientation towards individual support of children. With this in mind the following article tries to lay down the theoretical and empirical approaches of the planed project DiPoSa and discusses methodological foundations of the project. The discussion focuses on how assessment practices as well as assessment literacy can be promoted through a DBR research-design using video vignettes in the context of teacher education and qualification and rooting in a deepened science-practice partnership.

\section{Keywords}

Formative assessment; social studies and science in primary education; teacher education; video vignettes; Design-based-research

\section{Inhaltsverzeichnis}

1. Ausgangssituation und Problemstellung

2. Fachbezogene Lösungsansätze: Potentiale des Sachunterrichts nutzbar machen

3. Didaktisch-diagnostisches Handeln im inklusionsorientierten Sachunterricht - eine Standortbestimmung

3.1. Didaktisch-diagnostisches Handeln im Kontext aktueller Ansätze der Didaktik des Sachunterrichts

\subsection{Didaktisch-diagnostische Kompetenzen von Lehrkräften}

4. Entwicklungsforschung im Design-Based-Research-Design als Chance für inklusionsorientieren (Fach-) Unterricht

5. Potential des Einsatzes von Videovignetten für die Lehrer*innenaus- und -fortbildung

5.1. Videovignetten als realitätsnaher Zugang in der Theorie-Praxis-Verknüpfung

6. "Didaktisch-diagnostische Potentiale im inklusionsorientieren Sachunterricht" (DiPoSa) ein forschungsbasiertes Entwicklungsvorhaben

Literatur

Kontakt

Zitation

\section{Ausgangssituation und Problemstellung}

Bezogen auf den Diagnostikbegriff zeigt sich eine begriffliche Vielfalt, die sowohl produkt- als auch prozessorientierte Vorgehensweisen impliziert. Je nach Kontext und je nach fachlichen und disziplinären Prämissen werden unterschiedliche Schwerpunkte gesetzt. Unter einer eher allgemeinen, erziehungswissenschaftlichen Perspektive wird diagnostisches Handeln als Teil pädagogischen Handelns begriffen. Entsprechende Hinweise finden sich in bildungs- 
programmatischen Schriften und im schulpädagogischen Diskurs. So wird die Diagnosekompetenz von Lehrkräften in zentralen Empfehlungen der Lehrer*innenbildung (z.B. (Kultusministerkonferenz [KMK] \& Hochschulrektorenkonferenz [HRK], 2015), als Schlüsselkompetenz zur Realisierung einer individuellen Förderung der Schüler*innen und als notwendige Voraussetzung für schulische Inklusion herausgestellt. Diagnostisches Handeln wird somit als bedeutsamer Teil des pädagogischen Handelns von Lehrer*innen angesehen, denn es bedeutet, Lern-, Bildungs- und Entwicklungsprozesse zu gestalten, zu begleiten und zu unterstützen (Ricken \& Schuck, 2011, S. 110). Alle Lehrkräfte sollen „anschlussfähige allgemeinpädagogische und sonderpädagogische Basiskompetenzen für den professionellen Umgang mit Vielfalt in der Schule vor allem im Bereich der pädagogischen Diagnostik und der speziellen Förder- und Unterstützungsangebote entwickeln können“ (KMK \& HRK, 2015, S. 3). Unter den Bedingungen sehr heterogener Lerngruppen von Grundschulen sind somit alltägliches diagnostisches Handeln und die entsprechenden Kompetenzen, sowohl bei sämtlichen unterrichtlichen Entscheidungsprozessen als auch bei der Leistungsbeurteilung auf Seiten der Grundschullehrkräfte, ständig gefordert (Klemm, 2021, S. 56f). [1]

Entsprechende Ansatzpunkte sind bereits im Zuge schulischer Inklusion entwickelt worden. Beispielsweise wird ein Wandel (Simon \& Simon, 2014) konstatiert von eher traditionellen, statusdiagnostischen (d.h. formellen, normorientierten und individuumzentrierten Testverfahren, die sich medizinisch-psychologisch verorten lassen) hin zu förderdiagnostischen Vorgehensweisen, die für sich beanspruchen eher pädagogisch-psychologisch, formell und informell sowie systemisch angelegt zu sein und Aspekte von Lernen und Entwicklung in den Mittelpunkt zu stellen. Die Dualität des Begriffs der Förderdiagnostik impliziert, man würde bereits mit einer Förderintention diagnostizieren, was kritisch zu sehen ist, wenn es - u.U. gegenläufig zur pädagogischen Intention - vorrangig darum geht, Kinder als sonderpädagogisch förderbedürftig zu markieren, die Diagnostik seitens der sonderpädagogischen Profession durchgeführt wird und die entwickelten Förderpläne sich kaum in den folgenden didaktischen Unterrichtsplanungen wiederfinden (Moser Opitz, Pool Maag \& Labhart, 2019). Inklusionsorientierte Vorgehensweisen beanspruchen eine pädagogische Perspektive, die Ressourcen und Barrieren aufzeigt, bewusst lediglich Hypothesen formuliert und sowohl formell als auch informell angelegt ist, aber insbesondere einen verstehenden, qualitativen Zugang vorsieht (Moser Opitz et al., 2019), wobei strenggenommen zwischen Inklusion und Diagnostik auch eine Antinomie zu sehen ist. [2]

Konträr hierzu wird Diagnostik im schulischen Praxisfeld bisher primär als Aufgabenfeld sonderpädagogischer Profession in inklusiven Settings bestimmt (Melzer, Hillenbrand, Sprenger \& Hennemann, 2015). In der sonderpädagogischen Diagnostik, die häufig unmittelbar mit Selektionsentscheidungen verknüpft ist (Schäfer \& Rittmeyer, 2015), findet sich das Risiko des "Förderungs-Stigmatisierungs-Dilemmas“ (Boger \& Textor, 2016, S. 96), das durch aktuelle Inklusionsbestrebung eher rekontextualisiert denn gelöst wird. [3]

Trotz der umfassenden Forderung nach Inklusion wird die Selektions- und Allokationsfunktion sonderpädagogischer (Feststellungs-)Diagnostik nur unzureichend außer Kraft gesetzt, denn nach wie vor und teils zunehmend findet eine Kategorisierung von Kindern statt, was von Katzenbach (2015) als „systematische(n) Zufälligkeit der Vergabe des Labels ,Sonderpädagogischer Förderbedarf"“ (S.38) kritisiert wird. Dies zeigt sich in Teilen im erheblichen Anstieg bei der Vergabe dieses Labels: Zum einen hat sich der Anteil an Kindern mit einem Sonderpädagogischen Förderbedarf (SPF) in Deutschland in den letzten 20 Jahren von 4,4\% (1998) auf 7,4\% (2018) fast verdoppelt - wenn auch mit erheblichen Unterschieden je nach Region und Bundesland (KMK 2008; 2020). Zum anderen werden Kinder mit einem SPF in der BRD im internationalen Vergleich doppelt so häufig an Förderschulen unterrichtet, d.h. die Exklusionsquote stagniert auf einem hohen Niveau (European Agency, 2018; KMK, 2020). Dieser Aspekt ist auch aus einer Genderperspektive zu hinterfragen, denn die Gruppe der Kinder und Jugendlichen mit einem SPF ist zu zwei Dritteln männlich (Klemm, 2021, S. 38). [4] 
An der entsprechenden sonderpädagogischen Status- und Intelligenzdiagnostik ist schon vielfach Kritik geübt worden (Pfahl \& Powell, 2016; Kottmann, Miller \& Zimmer, 2018), ohne jedoch die fortbestehende Wirkmächtigkeit dieser langfristigen Etikettierung mit erheblichen Folgen für die Kinder zu reduzieren bzw. die professionellen Zuständigkeiten, die analog der Zwei-Gruppen-Theorie angelegt sind, zu hinterfragen. Dadurch, dass in NRW (wie auch in fast allen anderen Bundesländern) an einer expliziten Feststellung von SPF festgehalten wird (Gasterstädt, Kistner \& Adl-Amini, 2020), heben sich die Argumente gegen die Statusdiagnostik nicht auf. [5]

Auch die Förderdiagnostik bleibt in Bezug auf das genannte Dilemma nicht ausgespart, obwohl durch die enge Bindung an die Förderung eine ganz andere Form von Diagnostik behauptet und sie im Inklusionsdiskurs fest verankert ist (z.B. Heimlich \& Kahlert, 2012). Durch den untrennbaren Zusammenhang von Diagnostik und Förderung wird oftmals ein linearer Zusammenhang von Diagnose, Förderung und Lernerfolg suggeriert (Eberwein \& Knauer, 1998), der in der pädagogischen Praxis selten geben ist. Außerdem weisen einige Konzepte der Förderdiagnostik begrifflich ausschließlich der Lehrkraft die Aktivität des Förderns zu, während die Schüler*innen passiv bleiben (Schiefele, Streit \& Sturm, 2019). Es wird somit ein hierarchisches Verhältnis impliziert, das von impliziten Normalitätsvorstellungen geprägt ist (Textor, 2018), wodurch auch die Förderdiagnostik machttheoretisch bedeutsam bleibt und einem emanzipatorischen Bildungsbegriff entgegenstehen kann. Trautmann und Wischer (2011, S. 114) haben den semantischen Austausch der „bösen Selektionsdiagnostik“ gegen die "gute Förderdiagnostik" thematisiert und konstatieren, mit der Förderdiagnostik könne die Doppelfunktion von Diagnose zur Förderung und Auslese nicht außer Kraft gesetzt werden. [6]

Auch in unserem Konzept einer didaktischen Diagnostik können nicht sämtliche Funktionslogiken von Schule geleugnet und Dilemmata aufgelöst werden. Am Beispiel des relativ von Selektionszwängen befreiten Fachs Sachunterricht soll aber entwickelt werden, wie eine auf den Lernprozess der Kinder konzentrierte Diagnostik eine mögliche sonderpädagogische Engführung überwinden kann, indem sowohl die didaktischen Potentiale des Faches als auch ein breites grundschulpädagogisches Verständnis von Diagnostik, das auf Teilhabe, unterstützende Selbstdifferenzierung und kommunikativen Austausch (Bartnitzky, 2012) ausgerichtet ist, zu Grunde gelegt wird. Damit knüpfen wir explizit an das Plädoyer von Schuck (2004) an, diagnostische Überlegungen nicht losgelöst von pädagogischen Konzepten zu diskutieren, die sich unter einer fachdidaktischen Perspektive darstellen. Ziel des Projekts ist es deshalb, die eingangs vorgestellte, allgemeinpädagogische Perspektive auf Diagnostik spezifischer fachdidaktisch auszudifferenzieren und Konkretisierungen im Sinne einer lernunterstützenden Diagnostik zu entwickeln. [7]

Diese Argumente ziehen mehrere Folgerungen hinsichtlich einer systemischen Betrachtung der Situation und Problematik nach sich. Zum einen sind die institutionellen und professionellen Zuständigkeiten angesichts der negativen Effekte bei der Kategorisierung der Kinder zu hinterfragen. Zum anderen ist die Grundschule als Schule für alle Kinder ausdrücklich darin zu stärken, der gemeinsame Lernort für alle Kinder zu sein, was u.a. Konsequenzen für die Ausund Weiterbildung bzw. Qualifikation der Lehrkräfte mit sich bringt. Diese müssen auf eine alltagsintegrierte Diagnostik, die mit ihrem didaktischen Handeln verschränkt ist, stärker vorbereitet und dafür qualifiziert werden, die Kinder individualisiert und gleichzeitig mit Bezug auf einen gemeinsamen Lerngegenstand zu unterrichten. [8]

\section{Fachbezogene Lösungsansätze: Potentiale des Sachunterrichts nutzbar machen}

Das Fach Sachunterricht, das neben Deutsch und Mathematik ein Kern- bzw. Hauptfach der Grundschule ist, liefert aus seiner fachimmanenten Bedeutung zentrale Anknüpfungspunkte für eine ressourcenorientierte, didaktische Diagnostik (Prengel, 2016): Zum einen kommt dem Sachunterricht im Vergleich zu den Unterrichtsfächern Deutsch und Mathematik, für die eine Vielzahl an diagnostischen Verfahren vorliegt, quasi keine Selektionsfunktion zu. Bei einem vermuteten Förderbedarf beschreiben Lehrkräfte Lerndefizite in der Regel über die Fächer 
Deutsch und/oder Mathematik (Miller \& Kottmann, 2016). Das Fach Sachunterricht spielt in derartigen Beurteilungskontexten bislang (keine) bzw. eine untergeordnete Rolle. Damit bleiben auch mögliche Potentiale oder Kompetenzen der Kinder, jenseits mathematischer oder sprachlicher Grundfertigkeiten, im Fokus der Diagnostik zumeist unberücksichtigt. Zum anderen bietet der Sachunterricht durch seine grundsätzliche kindliche Lebensweltorientierung gepaart mit seiner vielperspektivischen Ausrichtung ein breites fachlich-inhaltliches und methodisches Spektrum (Gesellschaft für Didaktik des Sachunterrichts [GDSU], 2013), um alle Grundschulkinder über vielfältige unterrichtsmethodische Gestaltungsmöglichkeiten wie Handlungs- oder Problemorientierung (Schroeder \& Miller, 2017a) zu motivieren und an ihren individuellen Interessen und Könnenserfahrungen anzuknüpfen. Seitz und Simon (2021) verweisen auf die „Scharnierfunktion“ (S.6) des Faches, das zudem aus einer partizipativen Perspektive „als der Ort verstanden werden kann, an dem Fragen und Interessen von Kindern in divergenten Lebenslagen Raum erhalten“ (S.8). Daran anknüpfend sind die vielfältigen inhaltlichen und methodischen Chancen des Sachunterrichts zu nutzen, bewusst eine stärken- und ressourcenorientierte Sichtweise auf alle Kinder, d.h. im Sinne eines dialektischen Inklusionsverständnisses, auch Kinder in erschwerten Lern- und Entwicklungssituationen bzw. mit einem SPF (Lindmeier \& Lütje-Klose, 2015), einzunehmen. Aufbauend auf einer förderbezogenen individuellen Diagnostik gilt es diese fachlich und überfachlich im Sinne multikriterialer Zielerreichung in ihrer individuellen Persönlichkeitsentwicklung zu unterstützen. Dass eine multiple Zielerreichung (Blumberg, 2008) in einem inklusiven naturwissenschaftlichen Sachunterricht grundsätzlich möglich ist, zeigte sich in einer quasi-experimentellen Unterrichtsstudie (Blumberg \& Mester, 2018): Bei einer adaptiven, auf Kooperation und Ko-Konstruktion ausgerichteten Unterrichtsgestaltung, die den Kindern mit verschiedenen Unterstützungsangeboten ein individuelles Lernen am gemeinsamen Gegenstand ermöglichte, steigerten sich alle Kinder - auch Kinder mit einem SPF im Bereich Lernen - im Verstehen sowie in ihrer Motivation und Selbsteinschätzung (Blumberg \& Fromme, 2016) und erlebten sich als engagiert, kompetent und sozial eingebunden. [9]

Auf den skizzierten Potentialen des Sachunterrichts fußt die für dieses Forschungs- und Entwicklungsprojekt eingenommene Perspektive auf Diagnostik. Unter Nutzung der besonderen Charakteristika des Sachunterrichts wird mit dem Vorhaben in produktiver Weise auf das „Förderungs-Stigmatisierungs-Dilemma“ (Boger \& Textor, 2016) reagiert, indem eine lernprozessbegleitende Diagnostik, d.h. eine direkt in den Unterricht integrierte bzw. auf unterrichtliche Lehr-Lernprozesse bezogene Form der Diagnostik (Prengel, 2016), aus und für die Praxis erschlossen, forschungsbasiert konzeptualisiert und so für Aus- und Fortbildungszwecke nutzbar gemacht wird. [10]

\section{Didaktisch-diagnostisches Handeln im inklusionsorientierten Sachunterricht - eine Standortbestimmung}

Resultierend aus der eingangs formulierten Kritik an einer tendenziell defizitbezogenen und nicht unterrichtswirksamen sonderpädagogischen Diagnostik (z.B. Simon \& Simon, 2014) sowie den alternativ hierzu bestehenden theoretisch-konzeptionellen Überlegungen zu einer inklusionsorientierten, lernprozessbegleitenden bzw. didaktischen Diagnostik (vgl. Liebers \& Seifert, 2012; Prengel, 2016; Schiefele et al., 2019; Wocken, 2013) ergeben sich grundsätzlich hohe Anforderungen an eine diagnostisch-didaktische und förderbezogene Analyse individueller Lernwege (Schiefele et al., 2019). Schmidt (2020) etwa benennt hierzu auf Basis ihrer Untersuchung mit Grundschullehrkräften die Verwendung informeller und formeller Diagnoseverfahren, kooperativer Unterrichtselemente sowie den Anspruch an eine pädagogische Nutzung diagnostischer Informationen (auch Schütze, Souvignier \& Hasselhorn, 2018). Indem Strategien prozessbezogener Diagnostik bzw. des formativen Assessments auf die Verbesserung von Lehr-Lernprozessen auf die Mikroebene des Unterrichts abzielen, sieht Schmidt (2020) ein hohes Passungsverhältnis zu Prinzipien inklusionsorientierter Ansätze des Assessments und der Diagnostik (vgl. Liebers \& Seifert, 2012), obwohl eine Modifikation formativer Assessmentstrategien im Kontext inklusiver Lerngruppen für notwendig erachtet wird. Diese Adaption und Fortentwicklung bisheriger Strategien und Praktiken ist daher eine für uns leitende 
Zielperspektive. Deren Umsetzung im Sinne einer ressourcenorientieren und alltagsintegrierten lernprozessbegleitenden Diagnostik in der Primarstufe erscheint uns in besonderer Weise über das vielperspektivische Integrationsfach Sachunterricht mit seinen fachimmanenten Prinzipien ( Thomas, 2013; GDSU, 2013) - wie zuvor dargestellt - vielversprechend, indem die individuellen kindlichen Lernpotentiale als Ausgangspunkt für didaktisch-diagnostisches Handeln in den Fokus rücken. Daher ist zunächst zu fragen, welche Anknüpfungspunkte für didaktisch-diagnostisches Handels bereits in Ansätzen aus dem Fachdiskurs der Didaktik des Sachunterrichts bestehen. [11]

\subsection{Didaktisch-diagnostisches Handeln im Kontext aktueller Ansätze der Didaktik des Sachunterrichts}

In aktuellen inklusionsorientierten Ansätzen der Sachunterrichtsdidaktik finden im Kontext eines Umgangs mit Heterogenität bzw. der individuellen Förderung (Dumont, 2019) sowohl reformpädagogisch orientierte, offene Konzepte als auch Konzepte nach dem Modell des adaptiven Unterrichts ihren Niederschlag. Für das diagnostische Handeln zielen diese Konzepte - überspitzt formuliert - entweder eher auf ko-konstruktives Verstehen oder auf systematische Prozesse der Förderdiagnostik, wobei jedoch das Anknüpfen an Interessen und Vorstellungen der Schüler*innen eine Gemeinsamkeit beider Ansätze darstellt (Seitz \& Simon, 2018). [12]

Die zentrale Forderung nach einer Orientierung an Schüler*innenvorstellungen ist in der Sachunterrichtsdidaktik dabei fest verankert (GDSU, 2013; Schönknecht \& Maier, 2012; Skorsetz, Bonanati \& Kucharz, 2021). Wenngleich es mittlerweile eine elaborierte Forschungstradition zu Schüler*innenvorstellungen im Sachunterricht gibt (Murmann, 2013; Möller, 2018), liegen nur wenige Befunde dazu vor, ob und wie Lehrkräfte diese diagnostisch erfassen und bei ihren didaktischen Entscheidungen berücksichtigen. Einzelne Befunde zum Planungshandeln von Lehrkräften im Sachunterricht (Schroeder, 2020) legen den Schluss nahe, dass eine systematische Orientierung an Schüler*innenvorstellungen und individuellen Lernvoraussetzungen bei der Gestaltung des Unterrichts wenig Beachtung erfährt. Nichtsdestotrotz rückt die inklusionsorientierte Didaktik des Sachunterrichts (vgl. Pech, Schomaker \& Simon, 2019; Schroeder \& Miller, 2017a), die vielfältigen Zugangsweisen und Perspektiven der Kinder als Ausgangspunkt für subjektiv bedeutsames Lernen ins Zentrum, und zwar im Sinne eines individuellen und gleichzeitig gemeinsamen Lernens. [13]

Eine didaktische Diagnostik (vgl. Liebers, Maier, Prengel \& Schönknecht, 2013; Schönknecht \& Maier, 2012, Schroeder, 2016) soll (angehenden) Lehrkräften helfen, entsprechende anschlussfähige Entwicklungs- und Lernmöglichkeiten zu erkennen und zu realisieren, bei denen alle Kinder partizipativ eingebunden sind, indem ihre Fragen, Themen und Interessen aufgegriffen und ko-konstruktiv Gegenstand der Reflexion sind. Die Förderung der Kinder findet somit alltagsintegriert im didaktischen Handeln des Sachunterrichts statt. In den Forschungsarbeiten zum inklusiven Sachunterricht von Seitz (2005) wie auch Schomaker (2007) zu gestalteten offenen Lernumgebungen, wurde diese Doppelfunktion von Diagnostik und Didaktik in der Rekonstruktion individueller Lernwege aufgezeigt. Eigene Studien (z.B. Schroeder \& Miller, 2017b) zeigen, dass die Orientierung an Schüler*innenfragen ebenfalls eine Verbindungslinie zwischen einem schülerorientierten, offenen Unterricht und einem zielgerichteten, didaktischdiagnostischem Handeln darstellt. Schüler*innenfragen bilden einerseits den Ausgangspunkt für einen interessengeleiteten, subjektiv-bedeutsamen, partizipativen, forschend-entdeckenden Lernprozess und liefern gleichzeitig der Lehrkraft wertvolle Hinweise über das Vorwissen, kindliche Zugangs- und Denkweisen, Erfahrungshintergründe oder auch über Veränderungen im Gegenstandsverständnis (vgl. Brinkmann, 2019; Schroeder \& Miller, 2017a). [14]

Eine deutlich engere Ausrichtung des Unterrichtsangebots an den diagnostizierten Lernvoraussetzungen kennzeichnet den adaptiven Unterricht. Für den naturwissenschaftlichen Sachunterricht konnte die IGEL-Studie zur individuellen Förderung und adaptiven Lern-Gelegenheiten in der Grundschule (Decristan et al., 2017) positive Effekte formativen Assessments auf die Konzeptentwicklung der Schüler*innen nachweisen, während durch die beiden anderen 
Fördermethoden in Form der kognitiven Strukturierung und das tutorielle Lernen kein zusätzlicher Lerngewinn, auch im Vergleich zur Kontrollgruppe, stattfand. Das Treatment der lernbegleitenden Diagnostik erfolgte mittels diagnostischer Aufgaben, die am Ende einer Doppelstunde eingesetzt und von der Lehrkraft in der nächsten Stunde mit individuellem Feedback zurückgegeben wurden (Decristan et al., 2017). Dies schließt damit an generelle Befunde zur förderlichen Wirkung einer verstärkten unterrichtlichen Nutzung formativen Assessments auf den Lernerfolg der Schüler*innen (Black \& Wiliam, 1998; Hattie, 2013; Wylie et al., 2012; McMillian, Venable \& Varier, 2013) an. Mit Blick auf inklusives Lernen liefert die Studie von Decristan et al. (2017) aber noch ein weiteres zentrales Ergebnis: Die Gruppe der Kinder mit einem mangelnden Sprachverständnis in der Unterrichtssprache als Risikomerkmal für das naturwissenschaftliche Verständnis konnte besonders von einem Unterricht mit lernbegleitender Diagnostik aber ebenso von der kognitiven Strukturierung profitieren, der erwartbare Schereneffekt ließ sich dadurch verringern. Auch für das frühe naturwissenschaftliche Lernen im Elementarbereich wurden insbesondere für den sprachlichen Bereich im Projekt FinK (Formatives Assessment in der inklusiven naturwissenschaftlichen Bildung in der Kita) positive Effekte lernbegleitender Diagnostik anhand eines strukturierten Portfolios ermittelt, mit dem die pädagogischen Fachkräfte ausgehend von den Lernausgangslagen der Kinder abgestimmte, adaptive Förderentscheidungen zu naturwissenschaftlichen Bildungsangeboten treffen können (Hardy, Bürgermeister \& Leuchter, i. E.). Unabhängig davon in welcher Weise ein didaktischdiagnostisch begleiteter Umgang mit Heterogenität im Sachunterricht konzeptionell gedacht wird, geht dieser stets mit spezifischen Anforderungen an das professionelle Handeln und die damit verbundenen Kompetenzen der Lehrkräfte einher. Daher sollen folgend Implikationen diesbezüglicher Befunde diskutiert werden. [15]

\subsection{Didaktisch-diagnostische Kompetenzen von Lehrkräften}

Professionstheoretisch lässt sich diagnostische Kompetenz zentral dem fachdidaktischen Wissen zuordnen und beinhaltet ein Wissen über diagnostische Potentiale von Aufgaben ebenso wie Kenntnisse über Lernvoraussetzungen, Schüler*innenvorstellungen sowie Lernund Verständnisprozesse (Baumert \& Kunter, 2011). Für den Sachunterricht konnte die Forscher*innengruppe um Lange et al. (2012) positive Zusammenhänge zwischen dem fachdidaktischen Wissen von Lehrkräften und dem fachlichen Lernerfolg sowie motivational-selbstbezogenen Zieldimensionen auf Seiten der Schüler*innen nachweisen. Lehrkräfte mit einem guten bzw. sehr guten fachdidaktischen Wissen sind besser in der Lage, die kindlichen Vorerfahrungen und Präkonzepte aufzugreifen und sie in ein vernetztes Wissen gepaart mit positiven Lernerlebnissen zu überführen. Wenn damit eine lernprozessbegleitende Diagnostik an fachdidaktisches Wissen rückgekoppelt ist, muss es bedenkenswert sein, dass insbesondere Sonderpädagog*innen seltener über formale Qualifikationen im Sachunterricht verfügen und daher Grundschullehrkräfte an dieser Stelle häufiger auf fachdidaktische Expertise zurückgreifen können (Schroeder, i.E.). [16]

Vorliegende Befunde zur diagnostischen Kompetenz von Grundschullehrkräften (z.B. Kottmann et al., 2018; Südkamp, Kaiser \& Möller, 2012) generell deuten darauf hin, dass diese den Leistungsstand ihrer Schüler*innen realistisch und valide einschätzen, wohingegen dies bei übergreifenden lernrelevanten Fähigkeiten (Selbstkonzept, Intelligenz, Interessen) scheinbar nur bedingt gelingt (Spinath, 2005). Ebenso bestehen große Unterschiede in der jeweiligen diagnostischen Kompetenz zwischen einzelnen Lehrkräften (Südkamp et al., 2012). Neuere Untersuchungen zeigen positive Zusammenhänge zwischen Merkmalen diagnostischer Sensitivität (Behrmann \& Souvignier, 2013) bzw. Kompetenz (Brühwiler, 2014) und lernförderlichen Aktivitäten der Lehrkraft. Befunde zur diagnostischen Kompetenz von Sonderpädagog*innen stellen demgegenüber ein Forschungsdesiderat dar. [17]

Ungeachtet dessen werden die diagnostischen Kompetenzen von Grundschullehrkräften im Diskurs und z.B. auch seitens der HRK und KMK (2015) häufig unterschätzt. Dabei wird vernachlässigt, dass Grundschullehrkräfte in der Regel über elaborierte didaktische Qualifikationen in drei Unterrichtsfächern (i.d.R. Mathematik, Deutsch und ein weiteres Fach) verfügen 
und dass diagnostisches Handeln wie Beobachten, die Ermittlung individueller Lernstände etc. auch als genuine Elemente didaktischen Handelns angesehen werden können und bereits seit 2004 inhaltliche Ausbildungsschwerpunkte in allen Lehramtsstudiengängen sind. Studien zeigen zudem, dass Ergebnisse sonderpädagogischer Diagnostik nur selten in die Unterrichtsentwicklung einfließen und dass im Hinblick auf die Aufgabenwahrnehmung der Arbeitsbereichs Diagnostik in der Praxis nicht als exklusive Domäne der befragten Förderschullehrkräfte ausgewiesen wird (Moser \& Kropp, 2015). [18]

Einerseits wird deutlich, dass sowohl in der didaktisch-diagnostischen Expertise von Grundschullehrkräften bisher nicht hinreichend genutzte Potentiale zu vermuten sind, aber auch gerade eine Integrationsperspektive sonderpädagogischer Expertise mit Blick auf unterrichtswirksames diagnostisches Handeln noch nicht hinreichend elaboriert ist. Hier sehen wir diesbezüglich zentrale Ansatzpunkte für einen forschungsbasierten Entwicklungsansatz, der theoretisch-konzeptionell die skizzierten Lücken bearbeitet, aber ebenso der Dignität der Praxis (Dilger \& Euler, 2018) in der Ausgestaltung inklusiver Lernsettings Rechnung trägt. [19]

\section{Entwicklungsforschung im Design-Based-Research-Design als Chance für inklusionsorientieren (Fach-)Unterricht}

Um Bezug nehmend auf die dargestellten Desiderate hinsichtlich einer lernprozessbegleitenden didaktischen Diagnostik, die ressourcenorientiert und gleichzeitig förderbezogen angelegt und ausgerichtet ist, ein praxisorientiertes, implementierfähiges und gleichzeitig evidenzbasiertes Aus-, Fort-, und Weiterbildungsangebot für die Lehrer*innenbildung zu entwickeln, stellt der Forschungs- und Entwicklungsansatz des Design-Based-Research (DBR) (z.B. McKenney \& Reeves, 2019) eine geeignete Grundlage dar. Zur nachhaltigen Innovation (Bereiter, 2002) ist das besondere Auszeichnungsmerkmal dieses Ansatzes die von Anfang an kontinuierliche intensive Kooperation von Akteur*innen aus Praxis und Wissenschaft, die in einem gemeinsamen mehrfach wiederkehrenden zyklischen und iterativen Prozess der Analyse, Entwicklung, Erprobung, Evaluation und Weiterentwicklung (siehe Abbildung 1) „sowohl kontextualisierte Theorien des Lernens und Lehrens einschließlich Wissen zum Designprozess (theoretischer Output) als auch konkrete Verbesserungen für die Praxis und die Entfaltung innovativer Potentiale im Bildungsalltag (praktischer Output)" (Reinmann, 2005, S. 6; McKenney \& Reeves, 2019) hervorbringen. Das sogenannte Design, das im Rahmen des in diesem Beitrag vorgestellten Forschungs- und Entwicklungsprojekts (siehe Kapitel 6) entwickelt werden soll, zielt auf die Optimierung der Lehrer*innenaus-, fort- und -weiterbildung hinsichtlich der Ausbildung didaktischer Diagnose- und Förderkompetenzen in einem inklusionsorientierten Sachunterricht. Am Ende des mehrfach durchlaufenen Entwicklungszyklus (siehe Abbildung 1) wird ein modularisiertes Lernangebot für die Aus-, Fort- und Weiterbildung (zukünftiger) Grund- und Förderschullehrkräfte angestrebt, das ein Diagnose- und Beobachtungstool in Kombination mit Videovignetten (ausführlicher dazu siehe Kapitel 5) umfasst. Dazu fließen die gemeinsam aus realen reichhaltigen Sachunterrichtssituationen ermittelten Gelingens- bzw. Gestaltungsbedingungen für einen inklusionsorientierten auf die Potentiale der Kinder fokussierten Sachunterricht ein. In der von Anfang an DBR-typischen kontinuierlichen wechselseitigen Zusammenarbeit von schulischen und wissenschaftlichen Akteur*innen liegt dabei das Potential, ein tatsächlich für die Praxis passendes, praktikables und implementierbares Ausund Fortbildungsangebot für die Lehrer*innenbildung zu entwickeln, das die zuvor dargestellten Ansprüche einer ressourcenorientierten didaktischen Diagnostik erfüllt. [20] 


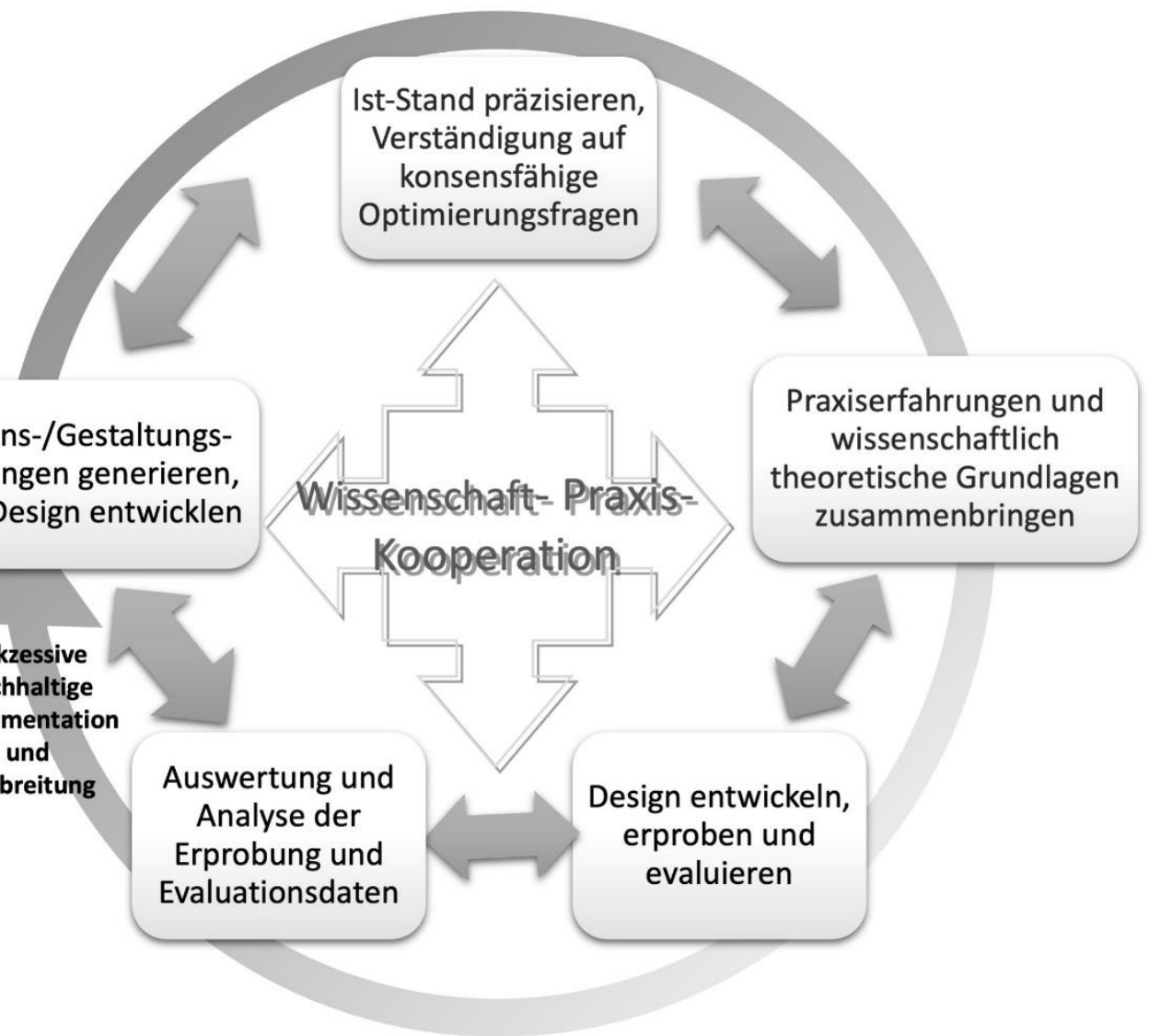

Abbildung 1: Design-Based-Research als praxisnahes Forschungs- und Entwicklungsdesign (eigene Abbildung)

Für die konkrete Umsetzung des DBR-Forschungs- und Entwicklungsdesigns (Bakker, 2019; McKenney \& Reeves, 2019; Reinmann, 2005) zur forschungsbasierten Entwicklung von Ausund Fortbildungsmodulen für die erste und dritte Phase der inklusionsorientierten Lehrer*innenbildung bedeutet das in stets enger Wissenschaft-Praxis-Kooperation (Dilger \& Euler, 2018) die folgenden Schritte (siehe Abbildung 1): Inklusions- und fortbildungserfahrene Sachunterrichtslehrkräfte und ein interdisziplinäres Wissenschaftsteam der Sachunterrichtsdidaktik sowie Grundschul- und Sonderpädagogik diskutieren gemeinsam die zuvor aufgezeigte Problematik rund um das „Förderungs-Stigmatisierungs-Dilemma“ (Boger \& Textor, 2016, S. 96) und einigen sich auf gemeinsam zu erreichende (Teil-)Ziele. Über das Zusammenbringen von wissenschaftlichen und theoretischen Grundlagen und Erfahrungen aus der Praxis werden erste Bausteine für das angestrebte modularisierte Lernangebot, d.h. ein erster Prototyp des Beobachtungs- und Diagnose-Tools sowie eine reichhaltige Sachunterrichtssequenz unter anderem als Grundlage zur Entwicklung von Videovignetten (siehe Kapitel 5), gestaltet, erprobt und evaluiert. Auf Grundlage von gemeinsam diskutierten Evaluationsergebnissen werden erste generelle Gelingens- bzw. Gestaltungsbedingungen herausgearbeitet und die Weiter- bzw. Neuentwicklung des modularisierten Lernangebots in einem weiteren Zyklus vorbereitet. Konkreter wird das Forschungs- und Entwicklungsdesign des Projektvorhabens in Kapitel 6 erläutert. Nachfolgend werden neuere Erkenntnisse und Potentiale der videobasierten Lehrer*innenaus-, fort-, und -weiterbildung dargelegt, die bei der Entwicklung der videobasierten Aus- und Fortbildungskonzeption für die Lehrer*innenbildung berücksichtigt werden. [21] 


\section{Potential des Einsatzes von Videovignetten für die Lehrerinnenaus- und - fortbildung}

Videofallbasiertes Lernen wird als gewinnbringender Ansatz angesehen, um die Entwicklung von Professionalität zu fördern, insbesondere mit Blick auf diagnostisch-analytische Fähigkeiten von Lehrkräften (Goeze, Hetfleisch \& Schrader, 2013). Besonderes Potential eröffnet ein videofallbasiertes Vorgehen einerseits zur Illustration vorab eingeführter Theoriebezüge, andererseits auch als Ausgangspunkt eines gemeinsamen, methodisch geleiteten Analyseprozesses (Bartel \& Roth, 2015). Ebenso kann über die fallbasierte, mediale Darstellungsform ein erhöhter Berufsfeldbezug in der akademischen Lehramtsausbildung hergestellt werden, welcher vielerorts mit dem Ziel einer intensiveren Theorie-Praxis-Verknüpfung gefordert wird (Kiel, Kahlert \& Haag, 2014). Insbesondere die spezifische Form der Fallarbeit anhand von Videovignetten bietet sowohl eine hohe Anschaulichkeit und Informationsdichte als auch eine ausreichende Realitätsnähe (Grewe \& Möller, 2020) und ermöglicht gleichsam eine Reduktion und Fokussierung auf bedeutsame Einzelaspekte, die jedoch die Komplexität der Unterrichtssituation als solche aufrechterhalten können. Empirisch belegt ist in diesem Kontext das besondere Potential des videofallbasierten Lernens gleichermaßen für angehende und erfahrene Lehrkräfte (Bartel \& Roth, 2015). Goeze (2010) stellt heraus, dass die Professionalisierungszugewinne sich innerhalb dieser beiden Gruppen jedoch grundlegend unterscheiden: Für Noviz*innen bietet die Fallarbeit die Möglichkeit einer grundlegenden Orientierung bzgl. der besonderen Komplexität von Unterrichtsszenarien. Mit Fokus auf die Entwicklung diagnostischer Kompetenzen ist diese Orientierungsleistung enorm relevant, da die Förderung der Kinder alltagsintegriert stattfinden soll (s. Kapitel 2.2) und so untrennbar mit der Analyse didaktischen Handelns im Unterricht verbunden ist. Expert*innen eröffnet die videofallbasierte Auseinandersetzung hingegen die Erarbeitung und Förderung eines übergeordneten Verständnisses von Praxissituationen. Insbesondere mit Fokus auf die diagnostischen Handlungsstrukturen von Grundschullehrkräften und mit Bezug auf übergreifende lernrelevante Fähigkeiten der Kinder (s. Kapitel 2.2) bieten videofallbasierte Fortbildungen eine Möglichkeit diese weiter zu elaborieren. [22]

Folgend wird genauer betrachtet, welche Potentiale das videofallbasierte Lernen zur Förderung einer alltagsintegrierten Diagnostik bietet und welche Anforderungen sich für den Einsatz in der Aus- und Weiterbildung von Lehrer*innen ergeben. [23]

\subsection{Videovignetten als realitätsnaher Zugang in der Theorie-Praxis-Verknüpfung}

Diagnostisches Handeln gilt als „fundamentaler Bestandteil der Unterrichtsplanung“ (Schroeder \& Miller, 2017a, S. 238), innerhalb dessen die „individuellen Lebenswelten, Interessen und Erfahrungen der Kinder" (Schroeder \& Miller, 2017a, S. 238) berücksichtigt werden müssen. Daraus ergibt sich mit Blick auf zu entwickelnde Aus- und Weiterbildungsmodule der Anspruch, dass diese didaktisch-diagnostisch sowohl auf der fachdidaktischen als auch auf der individuellentwicklungsbezogenen Ebene der Schüler*innen ergiebig sein müssen (Schroeder, 2016; Schroeder \& Miller, 2017a), mit dem Ziel der Förderung einer professionellen Unterrichtswahrnehmung und der damit einhergehenden analytischen Auseinandersetzung (Junker, Rauterberg, Möller \& Holodynski, 2020). Videovignetten werden in diesem Zusammenhang als geeigneter Zugang (Junker et al., 2020) und gegenüber textbasierten Falldarstellungen überlegen bewertet. Der Fokus liegt auf dem Wissensaufbau, der Ausprägung analytischer Fähigkeiten und einer gesteigerten Lern- und Transfermotivation, die sich in der Auseinandersetzung ergeben (Goeze, 2010). Weiterhin lassen sich durch den Einsatz von Videovignetten auch gegenüber originalem Praxiserleben weitreichende Vorteile ermitteln: Denn auch wenn Praxisphasen als Handlungs- und Resonanzfeld für theoretisch erworbene Wissensbestände gelten (Schwier \& Bulmahn, 2016) verdichten sich in der konkreten Praxis vielfältige und oftmals diffuse Ansprüche sowie explizit und implizit formulierte Erwartungen an die handelnden Akteur*innen, die sich insbesondere für Noviz*innen als problematisch in Bezug auf die Ausbildung professioneller Handlungskompetenzen auswirken können (Schwier \& Bulmahn, 2016). Ebenso wird schulische Praxis häufig nicht aus wissenschaftlich-theoriegeleiteter Perspektive erschlossen, sondern im Rahmen der individuellen Erfahrungsaufschichtung als „praktisch zu bewältigendes 
Handlungsfeld konstruiert" (Hedtke, 2007, S. 42). Konsequenzen dieser praktischen Auseinandersetzung und die damit konfligierende (Über-)Komplexität von Lehr-Lernsituationen begünstigen "die Aneignung mutmaßlich praktikabler Interpretations- und Handlungsmuster" (Schwier \& Bulmahn, 2016, S. 35), welche nicht ausreichend im Rahmen professionsorientierter Lernprozesse reflektiert werden, sondern vorliegende Common-Sense-Theorien bestätigen und manifestieren (Schwier \& Bulmahn, 2016). Insbesondere komplexe und vielschichtige Handlungsstrukturen, wie die Umsetzung einer alltagsintegrierten, lernprozessbegleitenden Diagnostik, sind im Kontext einer ausschließlich praktisch-intuitiven Erarbeitung schwer (weiter) zu entwickeln. [24]

Der Einsatz von videobasierten Falldarstellungen eröffnet in diesem Kontext die Möglichkeit mit einem hohen Praxisbezug die Komplexität von Unterrichtssituationen abzubilden und gleichzeitig nach dem Prinzip der Exemplarität den Fokus auf bedeutsame Einzelaspekte der Unterrichtssituation zu lenken. So kann eine Theorie-Praxis-Verzahnung gelingen mit dem Ziel theoriebasierte Reflexionen (Frey \& Buhl, 2018) mittels eines „authentischen, kontextspezifischen und motivierenden“ (Dannemann, Heeg \& Schanze, 2019, S. 76) Zugangs zu unterrichtsrelevanten Szenarien anzuregen. Innerhalb dieser kann eine Betrachtung der Unterrichtssituationen vollzogen werden, ohne einem Handlungsdruck oder Entscheidungszwang ausgesetzt zu sein (Krammer \& Reusser, 2005), was die Einnahme einer kritischen und distanzierten Perspektive auf die spezifische Situation erschwert (Krammer, 2014). [25]

Nach Goeze (2010) orientiert sich der Einsatz fallbasierter Videovignetten an den jeweiligen Qualifikationszielen und bedingt damit auch einen unterschiedlichen methodischen Zugang zum vorliegenden Material. Insbesondere mit Fokus auf verschiedene Qualifikationsziele muss somit eine Differenzierung bzgl. des analytischen Zugangs in entsprechenden Aus- und Weiterbildungsmodulen vorliegen. [26]

Neben der Berücksichtigung unterschiedlicher Qualifikationsziele existieren eine Vielzahl von empirischen Befunden über die grundlegende methodische Einbettung der Vignetten: In Anlehnung an Brophy (2004) kann anhand von Videofällen nur dann eine gewinnbringende Förderung professioneller Handlungsstrukturen erfolgen, wenn diese mittels gezielter Instruktionen analysiert werden. Demnach ist eine ungerichtete Betrachtung der Fälle nicht ausreichend zielführend (Goeze et al., 2013). Für einen gelingenden Einsatz ist jedoch nicht nur die Orientierung an den vorliegenden Qualifikationszielen und einem darauf abgestimmten methodischen Vorgehen relevant, sondern an die Form der Vignetten als solche ergeben sich ebenso entsprechende Qualitätsmerkmale. So erfordert es, dass die dargestellte Unterrichtsszenerie nicht nur einen spezifischen Aspekt, sondern multiple Aspekte beinhaltet, die in der anschließenden Auseinandersetzung und Diskussion von der Lerngruppe eingebracht und bearbeitet werden können (Goeze, 2010). Weiterhin spielt auch die Authentizität der dargestellten Unterrichtsszenen eine besondere Rolle in der Förderung diagnostischer Fähigkeiten (Goeze, Zottmann, Schrader \& Firscher, 2010). Dabei ist es grundsätzlich für den Lernprozess nicht relevant, ob die zu bearbeitenden Videofälle fremde oder eigene Unterrichtssequenzen enthalten (Grewe \& Möller, 2020). Kiel et al. (2014) formulieren dazu folgende Anforderungen, die einen guten „Fall für die Aus- und Weiterbildung von" (S.24) Lehrer*innen bestimmen: Ein erkennbarer Bezug zum Praxisfeld; „eine narrative Struktur“ (S.24); die „Handlungsmöglichkeiten nicht nur im Sinne eines Role-Takings, sondern auch im Sinne eines Role-Makings“ (S.24) eröffnet. Des Weiteren sollte der (Video-)Fall Kontingenzen und Spannungsfelder der Unterrichtspraxis widerspiegeln; zur Bearbeitung von „berufsfeldbezogenen Problemen“ (Kiel et al., 2014, S. 24) und zur Erarbeitung von Begründungszusammenhängen motivieren, die nicht ausschließlich „zweckrational, sondern auch wertrational oder dramaturgisch" (Kiel et al., 2014, S. 24) sein sollten. [27]

Im Folgenden wird das geplante Projekt vorgestellt, welches die verschiedenen theoretischen und methodischen Zugangsweisen, die im Verlauf dieses Beitrags erarbeitet wurden, ineinander überführt. [28] 


\section{6. „Didaktisch-diagnostische Potentiale im inklusionsorientieren Sachunterricht“" (DiPoSa) - ein forschungsbasiertes Entwicklungsvorhaben}

Wie den zuvor skizzierten theoretisch-konzeptionellen wie auch praxisbezogenen Entwicklungsbedarfen einer lernbegleitenden (fach-)didaktisch verorteten Diagnostik mit Blick auf Formate einer inklusionsorientierten Lehrer*innenbildung entsprochen werden kann, wird im nachfolgend vorgestellten Projektvorhaben „Didaktisch-diagnostische Potentiale des inklusionsorientierten Sachunterrichts (DiPoSa)" zusammengeführt. Vorangestellt ist die Ausgangshypothese, dass generell in der Grundschule und vor allem in der Sachunterrichtspraxis bereits didaktische Diagnosekompetenzen vorhanden sind, die durch eine entsprechende Aufbereitung systematisch in der Aus- und Weiterbildung weiterentwickelt werden können und dass das Fach Sachunterricht besondere Potentiale für einen ressourcenorientierten Blick auf Kinder ermöglicht. [29]

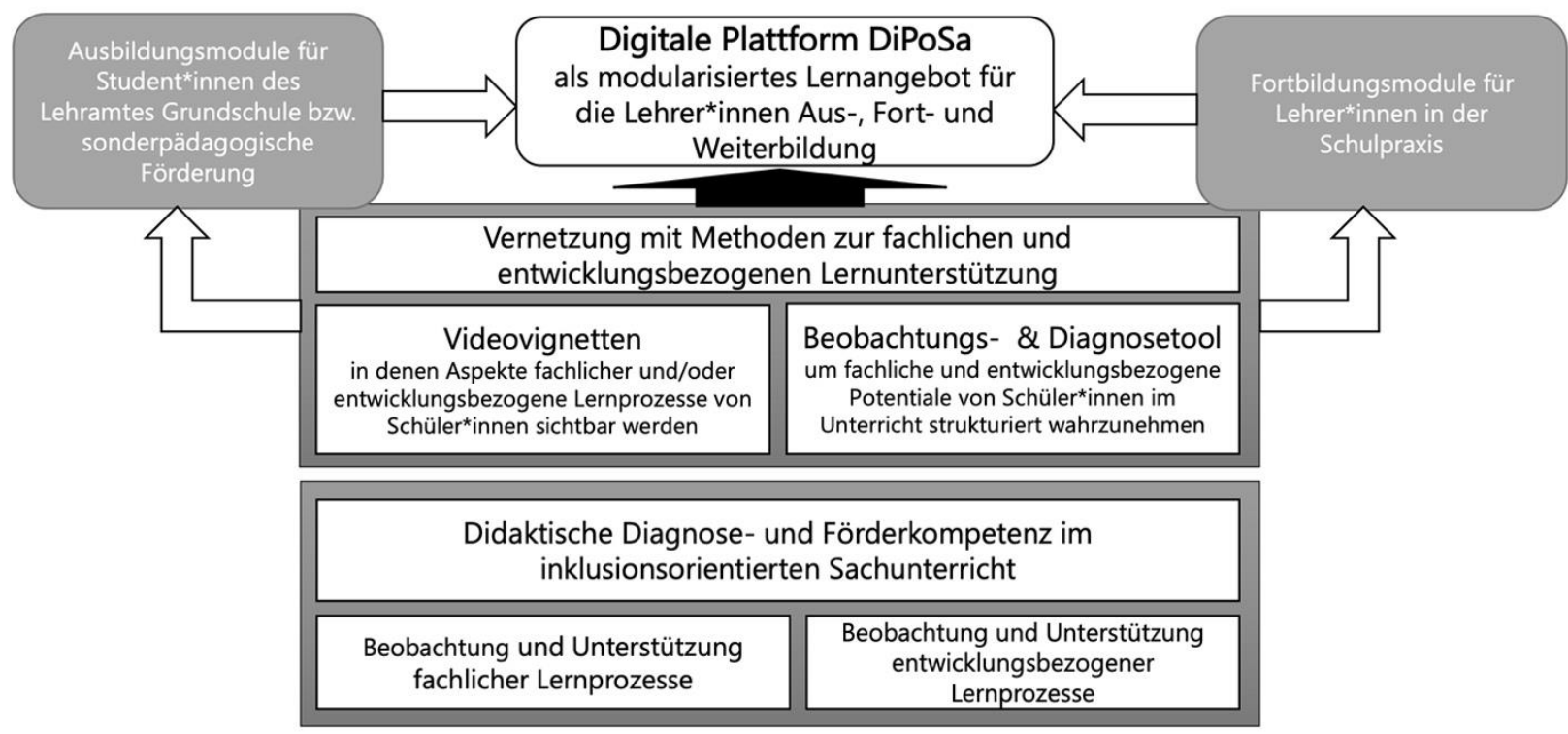

Abbildung 2: Der Aufbau des geplanten Projekts DiPoSa (eigene Darstellung)

Entsprechend dem zuvor dargestellten DBR-Ansatz sollen in dem Projekt über eine enge Wissenschaft-Praxis-Kooperation mit inklusions- und fortbildungserfahrenen Sachunterrichtslehrkräften im ersten Schritt didaktische Diagnose- und Förderkompetenzen identifiziert, konzeptualisiert und modelliert werden. In dieser ersten Projektphase der Ist-Stands- und Bedarfsanalyse finden dazu Gruppeninterviews mit erfahrenen Sachunterrichtslehrkräften zu ihrer bisherigen Praxis der Lernbegleitung und -unterstützung im Sachunterricht statt. Ziel ist hierbei nicht nur eine Bestandsaufnahme, sondern auch die Exploration möglicher Potentiale wie auch notwendiger Veränderungs- und Entwicklungsbedarfe. Ergänzt wird dies durch systematische Unterrichtsbeobachtungen bei den teilnehmenden Lehrkräften, um triangulativ erste Unterrichtssituationen hinsichtlich der vermuteten Potentiale bestimmen zu können. Nach dieser ersten Explorationsphase folgt ein erster Design- und Konstruktionsprozess. Die projektbeteiligten Lehrkräfte öffnen ihren Unterricht, um die benötigten Videografien anfertigen zu können. Bei der nachfolgenden gemeinsamen Auswahl und Verdichtung des Videomaterials zu ca. fünfminütigen Videovignetten, als Basis für die zu entwickelnden Aus- und Fortbildungsmodule, stehen Situationen im Fokus, in denen Lern- und Entwicklungsprozesse, aber auch Stärken und Barrieren einzelner Schüler*innen im Lernprozess sichtbar werden. Unter Einbezug der Expertise der beteiligten Lehrkräfte erfolgt eine erste Kategorisierung und Klassifikation der Videovignetten hinsichtlich unterschiedlicher Beobachtungs- und Analyseschwerpunkte zu verschiedenen fachlichen und entwicklungsbezogenen Facetten als Ausgangspunkte für die Förderung didaktisch-diagnostischer Analyse- und Reflexionskompetenzen in den geplanten Aus- und Fortbildungsmodulen. Eng verzahnt mit den möglichen Beobachtungs- und Analyse- 
schwerpunkten in den Vignetten erfolgt die Entwicklung eines Beobachtungs- und Diagnosetools, mittels dessen eine strukturierte und kriteriengeleitete Auseinandersetzung mit den didaktisch-diagnostisch ergiebigen Situationen in den Vignetten erfolgen kann. Dieses Tool dient dabei einerseits als Unterstützungsstruktur für den Aus- und Fortbildungskontext wie auch transferorientiert für die spätere praktische Arbeit im eigenen Unterricht. Das Tool soll Indikatoren gestützt helfen vorhandene Ressourcen oder Barrieren in den fachlichen und überfachlichen wie entwicklungsbezogenen Lernprozessen der Schüler*innen (Schroeder, 2019; Wocken, 2013) systematisch zu erfassen, zu bewerten und im Sinne eines Förderkreismodells (Schönknecht \& Maier, 2012) daraus resultierend individuelle Unterstützungsbedarfe ableiten zu können. Um die geforderte enge Verschränkung (siehe Kap. 2) von diagnostischem und didaktischem Handeln zu gewährleisten, werden zusätzlich für jede Videovignette Vorschläge zur fachlichen wie entwicklungsbezogenen Lernunterstützung der Schüler*innen im Sinne wirksamer Scaffoldingtechniken (Möller, 2016) ausgearbeitet. Diese sind als Anregung und Diskussionsgrundlage für die Arbeit in den Aus- und Fortbildungsmodulen gedacht. [30]

Im zweiten Design- und Konstruktionsschritt werden basierend auf den referierten Befunden zu wirksamen Formaten videogestützter Lehrer*innenaus- und -fortbildung entsprechende Ausund Fortbildungsmodule konzipiert. Zielgruppe sind einerseits Studierende des Lehramtes an Grundschulen bzw. für sonderpädagogische Förderung und andererseits berufserfahrene Grundschullehrkräfte und Sonderpädagog*innen an inklusiven Grundschulen. Die Erprobung auf Studierendenebene erfolgt in Seminaren zur Diagnose und Förderung im Rahmen der Bachelor- bzw. Masterstudiengänge an den Universitäten Bielefeld und Paderborn in drei Erprobungsdurchgängen. In enger Kooperation mit den Kompetenzteams für Lehrer*innenfortbildung in den Schulamtsbezirken Bielefeld, Gütersloh, Herford und Paderborn finden parallel Fortbildungsveranstaltungen für Lehrkräfte statt. Evaluativ werden dabei sowohl Wirkungen auf Ebene des individuellen Professionalisierungsprozesses, insbesondere hinsichtlich inklusionsbezogener Selbstwirksamkeitsüberzeugungen und förderbezogener Diagnosekompetenz (Assessment Literacy), überprüft, wie auch Implementationsbedingungen der Modulkonzeption als solche untersucht. Auf Basis der Ergebnisse der prozessbegleitenden Konzeptevaluation werden die Module modifiziert und finalisiert. Die abschließend manualisiert vorliegenden Aus- und Fortbildungsmodule werden zum Projektende über eine Onlineplattform interessierten Kolleg*innen aus Wissenschaft und Praxis allgemein zugänglich gemacht. [31]

Insgesamt soll das Projekt DiPoSa somit einen Beitrag dazu leisten, Lehrer*innen in ihrer inklusiven Schulpraxis zu qualifizieren und zu stärken, einen ressourcenorientierten Blick auf Kinder und deren individuelle Lernvoraussetzungen und -verläufe einzunehmen, ohne dass die kindlichen Lernbedarfe zur Legitimierung von Ressourcen oder zur Etikettierung der Schüler*innen führen. [32]

\section{Literatur}

Bakker, A. (2019). Design Research in Education. London, New York: Routledge.

Bartel, M.-E. \& Roth, J. (2015). Diagnostische Kompetenz durch Videovignetten fördern. In F. Caluori, H. Linneweber-Lammerskitten \& C. Streit (Hrsg.), Beiträge zum Mathematikunterricht (S. 1033-1036). Münster: WTM-Verlag.

Bartnitzky, H. (2012). Fördern heißt Teilhabe. In H. Bartnitzky \& H. Brügelmann (Hrsg.), Individuell fördern - Kompetenzen stärken in der Eingangsstufe (S. 6-36). Frankfurt am Main: Strube Druck.

Baumert, J. \& Kunter, M. (2011). Das Kompetenzmodell von COACTIV. In M. Kunter, J. Baumert, W. Blum, U. Klusmann, S. Krause \& M. Neubrand (Hrsg.), Professionelle Kompetenz von Lehrkräften. Ergebnisse des Forschungsprogramms COACTIV (S. 29-53). Münster: Waxmann. 
Behrmann, L. \& Souvignier, E. (2013). The Relation Between Teachers' Diagnostic Sensitivity, their Instructional Activities, and their Student's Achievement Gains in Reading. Zeitschrift für Pädagogische Psychologie, 27(4), 283-293. doi:10.1024/1010-0652/a000112

Bereiter, C. (2002). Design research for sustained innovation. Cognitive Studies, Bulletin of the Japanese Cognitive Science Society, 9(3), 321-327. doi:10.11225/jcss.9.321

Black, P. \& Wiliam, D. (1998). Assessment and Classroom Learning. Assessment in Education: Principles, Policy \& Practice, 5(1), 7-74. doi:10.1080/0969595980050102

Blumberg, E. (2008). Multikriteriale Zielerreichung im naturwissenschaftsbezogenen Sachunterricht der Grundschule. Eine Studie zum Einfluss von Strukturierung in schülerorientierten Lehr-Lernumgebungen auf das Erreichen kognitiver, motivationaler und selbstbezogener Zielsetzungen. (Dissertation). Westfälischen Wilhelms-Universität, Münster. Verfügbar unter: https://miami.uni-muenster.de/Record/d767660e-c351-46ca9828-7075ef173a3c

Blumberg, E. \& Fromme, T. (2016). Fostering inclusive learning in adaptive learning environments for primary science education. In J. Lavonen, K. Jutti, J. Lampiselkä, A. Uitto \& K. Hahl (Hrsg.), Electronic Proceedings of the ESERA 2015 Conference. Science education research: Engaging learners for a sustainable future (Bd. 16, S. 2748-2759).

Blumberg, E. \& Mester, T. (2018). Kognitv inhaltsbezogenes Lernen im inklusiven naturwissenschaftlich-technischen Sachunterricht.im Diskurs. In S. Miller, B. Holler-Nowitzki, B. Kottmann, S. Lesemann, B. Letmathe-Henkel, N. Meyer et al. (Hrsg.), Profession und Disziplin. Grundschulpädagogik im Diskurs (Jahrbuch Grundschulforschung, Bd. 22, S. 178184). Wiesbaden: Springer.

Boger, M. \& Textor, A. (2016). Das Förderungs-Stigmatisierungs-Dilemma. Oder: Der Effekt diagnostischer Kategorien auf die Wahrnehmung durch Lehrkräfte. In B. Amrhein (Hrsg.), Diagnostik im Kontext inklusiver Bildung (S. 79-97). Bad Heilbrunn: Klinkhardt.

Brinkmann, V. (2019). Fragen stellen an die Welt. Baltmannsweiler: Schneider.

Brophy, J. (2004). Using video in a teacher education. Amsterdam: Elsevier.

Brühwiler, C. (2014). Adaptive Lehrkompetenz und schulisches Lernen. Effekte handlungssteuernder Kognitionen von Lehrpersonen auf Unterrichtsprozesse und Lernergebnisse der Schülerinnen und Schüler. Münster: Waxmann.

Dannemann, S., Heeg, J. \& Schanze, S. (2019). Fallbasierte Förderung der Diagnose- und Planungsfähigkeiten von Lehramtsstudierenden. Lernen mit Videovignetten in der Biologieund Chemiedidaktik. In T. Leuders, E. Christophel, M. Hemmer, F. Korneck \& P. Labudde (Hrsg.), Fachdidaktische Forschung zur Lehrerbildung (Fachdidaktische Forschung, Bd. 11, S. 75-86). Münster: Waxmann.

Decristan, J., Hardy, I., Klieme, E., Büttner, G., Hertel, S., Kunter, M. et al. (2017). Individuelle Förderung und adaptive Lerngelegenheiten im Grundschulunterricht. In U. Hartmann, M. Hasselhorn \& A. Gold (Hrsg.), Entwicklungsverläufe verstehen - Individuelle Förderung wirksam gestalten. Forschungsergebnisse des Frankfurter IDeA-Zentrums (S. 312-326). Stuttgart: Kohlhammer.

Dilger, B. \& Euler, D. (2018). Wissenschaft und Praxis in der gestaltungsorientierten Forschung - ziemlich beste Freunde? Verfügbar unter: http://www.bwpat.de/ausgabe33/dilger_euler_ bwpat33.pdf

Dumont, H. (2019). Neuer Schlauch für alten Wein? Eine konzeptuelle Betrachtung von individueller Förderung im Unterricht. Zeitschrift für Erziehungswissenschaft, 22, 249-277. doi:10.1007/s11618-018-0840-0

Eberwein, H. \& Knauer, S. (1998). Handbuch Lernprozesse verstehen. Wege einer neuen (sonder-)pädagogischen Diagnostik. Weinheim: Beltz.

European Agency. (2018). European Agency Statistics on inclusive Education. 2016 Dataset Cross-Country Report. Verfügbar unter: https://www.european-agency.org/resources /publications/european-agency-statistics-inclusive-education-2018-dataset-cross-country 
Frey, A. \& Buhl, H. (2018). Professionalisierung von Grundschullehrkräften - wissenschaftlich fundiert, praxisorientiert und reflexionsbasiert. Zeitschrift für Grundschulforschung, 11, 199213.

Gasterstädt, J., Kistner, A. \& Adl-Amini, K. (2020). Die Feststellung sonderpädagogischen Förderbedarfs als institutionelle Diskriminierung? Eine Analyse der schulgesetzlichen Regelungen. Zeitschrift für Inklusion online, (4). Verfügbar unter: https://www.inklusiononline.net/index.php/inklusion-online/article/view/551

Gesellschaft für Didaktik des Sachunterrichts. (2013). Perspektivrahmen Sachunterricht (2. Aufl.). Bad Heilbrunn: Klinkhardt.

Goeze, A. (2010). Was ist ein guter Fall? In J. Schrader, R. Hohmann \& S. Hartz (Hrsg.), Mediengestützte Fallarbeit (S. 125-145). Bielefeld: Bertelsmann. doi:10.3278/6004103w

Goeze, A., Hetfleisch, P. \& Schrader, J. (2013). Wirkungen des Lernens mit Videofällen bei Lehrkräften. Zeitschrift für Erziehungswissenschaft, 16, 79-113. doi:10.1007/s11618-0130352-x

Goeze, A., Zottmann, J., Schrader, J. \& Firscher, F. (2010). Instructional support for case-based learning with digital videos. In D. Gibson \& B. Didge (Hrsg.), Proceedings of the Society for Information technology \& Teacher Education International Confrence (S. 1098-1104). Chesapeake: AACE.

Grewe, O. \& Möller, K. (2020). Die professionelle Unterrichtswahrnehmung von sprachsensiblen Maßnahmen im Sachunterricht der Grundschule fördern. HLZ. doi:10.4119/hlz-254 7

Hardy, I., Bürgermeister, A. \& Leuchter, M. (i. E.). Portfolios in der frühen naturwissenschaftlichen Bildung: Bedeutung für die Sprachförderung in inklusiven Kontexten. In $\mathrm{E}$. Blumberg, C. Niederhaus \& A. Mischendahl (Hrsg.), Sprachbildend Lernen und Lehren im, vor und nach dem Sachunterricht. Stuttgart: Kohlhammer.

Hattie, J. (2013). Lernen sichtbar machen. Überarbeitete deutschsprachige Ausgabe von "Visible Learning". Baltmannsweiler: Schneider.

Hedtke, R. (2007). Das Studium als vorübergehende Unterbrechung der Schulpraxis. Anmerkung zur geschlossenen Welt der Lehrerausbildung. In F. Kostrzewa (Hrsg.), Lehrerbildung im Diskurs (S. 25-89). Berlin: Lit-Verlag.

Heimlich, U. \& Kahlert, J. (2012). Inklusion in Schule und Unterricht. Stuttgart: Kohlhammer.

Junker, R., Rauterberg, T., Möller, K. \& Holodynski, M. (2020). Videobasierte Lehrmodule zur Förderung der professionellen Wahrnehmung von heterogenitätssensiblem Unterricht. $H L Z$. doi:10.4119/hlz-2554

Katzenbach, D. (2015). De-Kategorisierung inklusive? Über Risiken und Nebenwirkungen des Verzichts auf Etikettierungen. In C. Huf \& I. Schnell (Hrsg.), Inklusive Bildung in Kita und Grundschule (S. 33-55). Stuttgart: Kohlhammer.

Kiel, E., Kahlert, J. \& Haag, L. (2014). Was ist ein guter Fall für die Aus- und Weiterbildung von Lehrerinnen und Lehrern? Beiträge zur Lehrerinnen- und Lehrerbildung, 32(1), 21-33.

Klemm, K. (2021). Inklusion in Deutschlands Schulen. Entwicklungen - Erfahrungen Erwartungen. Weinheim: Beltz Juventa.

Kottmann, B., Miller, S. \& Zimmer, M. (2018). Macht Diagnostik Selektion? Zeitschrift für Grundschulforschung, 11(1), 23-38. doi:10.1007/s42278-018-0008-2

Krammer, K. (2014). Fallbasiertes Lernen mit Unterrichtsvideos in der Lehrerinnen- und Lehrerbildung. Beiträge zur Lehrerinnen- und Lehrerbildung, 32(2), 164-175.

Krammer, K. \& Reusser, K. (2005). Unterrichtsvideos als Medium der Aus- und Weiterbildung von Lehrpersonen. Beiträge zur Lehrerbildung, 23(1), 35-50.

Kultusministerkonferenz. (2008). Sonderpädagogische Förderung in Schulen 1997 bis 2006. Bonn/Berlin: Statistische Veröffentlichungen, Dokumentation Nr. 185.

Kultusministerkonferenz. (2020). Sonderpädagogische Förderung in Schulen 2009 bis 2018. Berlin: Statistische Veröffentlichungen, Dokumentation Nr. 223. 
Kultusministerkonferenz \& Hochschulrektorenkonferenz. (2015). Lehrerbildung für eine Schule der Vielfalt. Verfügbar unter: https://www.hrk.de/fileadmin/_migrated/content_uploads/HRKKMK-Empfehlung_Inklusion_in_LB_032015.pdf

Lange, K., Kleickmann, T., Tröbst, S. \& Möller, K. (2012). Fachdidaktisches Wissen von Lehrkräften und multiple Ziele im naturwissenschaftlichen Sachunterricht. Zeitschrift für Erziehungswissenschaft, 15(1), 55-75. doi:10.1007/s11618-012-0258-Z

Liebers, K., Maier, P., Prengel, A. \& Schönknecht, G. (2013). Pädagogische Diagnostik und Lernwege von Kindern im inklusiven Sachunterricht. In S. Wittkowske \& K. v. Maltzahn (Hrsg.), Lebenswirklichkeit und Sachunterricht. Erfahrungen - Ergebnisse - Entwicklungen (S. 48-62). Bad Heilbrunn: Klinkhardt.

Liebers, K. \& Seifert, C. (2012). Assessmentkonzepte für die inklusive Schule - eine Bestandsaufnahme. Zeitschrift für Inklusion online, (3). Verfügbar unter: https://www.inklusiononline.net/index.php/inklusion-online/article/view/44

Lindmeier, C. \& Lütje-Klose, B. (2015). Inklusion als Querschnittsaufgabe in der Erziehungswissenschaft. Erziehungswissenschaft, (51), 7-16.

McKenney, S. \& Reeves, T. C. (2019). Conducting Educational Design Research (2. Aufl.). London, New York: Routledge.

McMillian, J. H., Venable, J. C. \& Varier, D. (2013). Studies of the Effect of Formative Assessment on Student Achievement: So Much More is Needed. Practical Assessment, Research, and Evaluation, 18(2), 1-15. doi:10.7275/tmwm-7792

Melzer, C., Hillenbrand, C., Sprenger, D. \& Hennemann, T. (2015). Aufgaben von Lehrkräften in inklusiven Bildungssystemen - Review internationaler Studien. Erziehungswissenschaft, 26(51), 61-80. doi:10.3224/ezw.v26i2.21070

Miller, S. \& Kottmann, B. (2016). „Und dann war das auch noch so ein kleines, zartes Persönchen «. Grundschullehrkräfte im Entscheidungsdilemma zwischen Fördern und Selektieren. In B. Amrhein (Hrsg.), Diagnostik im Kontext inklusiver Bildung (S. 154-167). Bad Heilbrunn: Klinkhardt.

Möller, K. (2016). Bedingungen und Effekte qualitätsvollen Unterrichts - ein Beitrag aus fachdidaktischer Perspektive. In N. McElvany \& W. W. Bos (Hrsg.), Bedingungen und Effekte guten Unterrichts (S. 43-64). Münster: Waxmann.

Möller, K. (2018). Die Bedeutung von Schülervorstellungen für das Lernen im Sachunterricht. In M. Adamina, M. Kübler, K. Kalcsics, S. Bietenhard \& E. Engeli (Hrsg.), „Wie ich mir das denke und vorstelle..." Vorstellungen von Schülerinnen und Schülern zu Lerngegenständen des Sachunterrichts und des Fachbereichs Natur, Mensch, Gesellschaft (S. 35-50). Bad Heilbrunn: Klinkhardt

Moser, V. \& Kropp, A. (2015). Kooperation in Inklusiven Settings (KIS) - Vorarbeiten zu einem Kompetenzstrukturmodell sonderpädagogischer Lehrkräfte. In T. H. Häcker \& M. Walm (Hrsg.), Inklusion als Entwicklung. Konsequenzen für Schule und Lehrerbildung (S. 185212). Bad Heilbrunn: Klinkhardt.

Moser Opitz, E., Pool Maag, S. \& Labhart, D. (2019). Förderpläne: Instrument zur Förderung oder "bürokratisches Mittel"? Eine empirische Untersuchung zum Einsatz von Förderplänen. Empirische Sonderpädagogik, 3, 210-224.

Murmann, L. (2013). Dreierlei Kategoriebildung zu Schülervorstellungen im Sachunterricht? Text, Theorie und Variation - Ein Versuch, methodische Parallelen und Herausforderungen bei der Erschließung von Schülervorstellungen aus Interviewdaten zu erfassen, (19). Verfügbar unter: http://www.widerstreit-sachunterricht.de/

Pech, D., Schomaker, C. \& Simon, T. (2019). Inklusion im Sachunterricht. Perspektiven der Forschung. Bad Heilbrunn: Klinkhardt.

Pfahl, L. \& Powell, J. (2016). „Ich hoffe sehr, sehr stark, dass meine Kinder mal eine normale Schule besuchen können“. Pädagogische Klassifikation und ihre Folgen für die (Selbst)Positionierung von Schüler/innen. Zeitschrift für Pädagogik, 58-74. 
Prengel, A. (2016). Didaktische Diagnostik als Element alläglicher Lehrerarbeit - "Formatives Assessment" im inklusiven Unterricht. In B. Amrhein (Hrsg.), Diagnostik im Kontext inklusiver Bildung (S. 49-63). Bad Heilbrunn: Klinkhardt.

Reinmann, G. (2005). Innovation ohne Forschung? Ein Plädoyer für den Design-Based Research-Ansatz in der Lehr-Lernforschung. Unterrichtswissenschaft, 33(1), 52-69.

Ricken, G. \& Schuck, K. D. (2011). Pädagogische Diagnostik und Lernen. In A. Kaiser, P. Wachtel, B. Werner \& D. Schmetz (Hrsg.), Behinderung, Bildung, Partizipation (S. 110-119). Stuttgart: Kohlhammer.

Schäfer, H. \& Rittmeyer, C. (2015). Inklusive Diagnostik. In H. Schäfer \& C. Rittmeyer (Hrsg.), Handbuch Inklusive Diagnostik (S. 103-134). Weinheim: Beltz.

Schiefele, C., Streit, C. \& Sturm, T. (2019). Pädagogische Diagnostik und Differenzierung in der Grundschule. München: Reinhardt.

Schmidt, C. (2020). Formatives Assessment in der Grundschule. Konzept, Einschätzungen der Lehrkräfte und Zusammenhänge. Wiesbaden: Springer VS. doi:10.1007/978-3-658-26921-0

Schomaker, C. (2007). Der Faszination begegnen: Ästhetische Zugangsweisen im Sachunterricht. Oldenburg: DIZ.

Schönknecht, G. \& Maier, P. (2012). Diagnose und Förderung im Sachunterricht. Kiel: IPN.

Schroeder, R. (i.E.). „(Un-)gestört bei der Sache? - eine explorative Vergleichsstudie zur Praxis des Sachunterrichts aus Perspektive von Lehrkräften im Gemeinsamen Unterricht und an Förderschulen mit dem Förderschwerpunkt emotionale und soziale Entwicklung in Nordrhein-Westfalen. Bad Heilbrunn: Klinkhardt.

Schroeder, R. (2016). Diagnostik im inklusiven Sachunterricht - Zwischen Fachbezug und Lebenswelt. In H. Giest, T. Goll \& A. Hartinger (Hrsg.), Sachunterricht - zwischen Kompetenzorientierung, Persönlichkeitsentwicklung, Lebenswelt und Fachbezug (Probleme und Perspektiven des Sachunterrichts, Bd. 26, S. 75-83). Bad Heilbrunn: Klinkhardt.

Schroeder, R. (2019). Inklusiver Unterricht - didaktische Herausforderungen in heterogenen Lerngruppen. In R. Markowetz (Hrsg.), Sonderpädagogen an inklusiven Schulen. Rollen, Aufgaben und Kompetenzen in der Praxis (S. 221-350). Stuttgart: Raabe.

Schroeder, R. (2020). Inklusiver Sachunterricht zwischen Kind- und Materialorientierung Mediennutzung und Motive der Medienauswahl im Fokus einer explorativen Lehrkräftebefragung. Zeitschrift für Grundschulforschung, 13, 81-97. doi:10.1007/s42278019-00070-7

Schroeder, R. \& Miller, S. (2017a). Sachunterrichtsdidaktik und Inklusion. In F. Hellmich \& E. Blumberg (Hrsg.), Inklusiver Unterricht in der Grundschule (S. 231-247). Stuttgart: Kohlhammer.

Schroeder, R. \& Miller, S. (2017b). Schülerfragen im Sachunterricht am Beispielthema "Brücken - und was sie stabil macht". In H. Giest, A. Hartinger \& S. Tänzer (Hrsg.), Vielperspektivität im Sachunterricht (Schriftenreihe der Gesellschaft für Didaktik des Sachunterrichtes e.V, Bd. 27, S. 185-192). Bad Heilbrunn: Klinkhardt.

Schuck, K. D. (2004). Zur Bedeutung der Diagnostik bei der Begleitung von Lern- und Entwicklungsprozessen. Zeitschrift für Heilpädagogik, (8), 350-360.

Schütze, B., Souvignier, E. \& Hasselhorn, M. (2018). Stichwort - Formatives Assessment. Zeitschrift für Erziehungswissenschaft, 21(4), 697-715. doi:10.1007/s11618-018-0838-7

Schwier, V. \& Bulmahn, C. (2016). „Miniaturen sozialwissenschaftsdidaktischer Professionalität“ - Über Wissen und Können in unterrichtlichen Praxisphasen. Zeitschrift für Didaktik der Gesellschaftswissenschaften, 7(2), 32-53.

Seitz, S. (2005). Zeit für inklusiven Sachunterricht (Basiswissen Grundschule, Bd. 18). Baltmannsweiler: Schneider Hohengehren.

Seitz, S. \& Simon, T. (2018). Grundlagen und Prinzipien diagnostischen Handelns im inklusiven Sachunterricht. In D. Pech, C. Schomaker \& T. Simon (Hrsg.), Sachunterrichtsdidaktik \& Inklusion. Ein Beitrag zur Entwicklung (S. 80-95). Baltmannsweiler: Schneider. 
Seitz, S. \& Simon, T. (2021). Inklusive Bildung und Fachdidaktik in Grundschulen. Erkenntnisse, Reflexionen und Perspektiven. Zeitschrift für Grundschulforschung, 14, 1-14. doi:10.1007/s42278-020-00096-2

Simon, J. \& Simon, T. (2014). Inklusive Diagnostik - Wesenszüge und Abgrenzung von traditionellen "Grundkonzepten « diagnostischer Praxis. Eine Diskussionsgrundlage. Zeitschrift für Inklusion.

Skorsetz, N., Bonanati, M. \& Kucharz, D. (2021). Diversität und soziale Ungleichheit als Herausforderung an die Grundschule. In N. Skorsetz, M. Bonanati \& D. Kucharz (Hrsg.), Diversität und soziale Ungleichheit (S. 2-7). Wiesbaden: Springer. doi:10.1007/978-3-65827529-7

Spinath, B. (2005). Akkuratheit der Einschätzung von Schülermerkmalen durch Lehrer und das Konstrukt der diagnostischen Kompetenz. Zeitschrift für Pädagogische Psychologie, 19(1/2), 85-95.

Südkamp, A., Kaiser, J. \& Möller, J. (2012). Accuracy of Teachers' Judgments of Students' Academic Achievement: A Meta-Analysis. Journal of Educational Psychology, 104(3), 743762. doi:10.1037/a0027627

Textor, A. (2018). Einführung in die Inklusionspädagogik. Bad Heilbrunn: Klinkhardt.

Thomas, B. (2013). Der Sachunterricht und seine Konzeptionen. Historische und aktuelle Entwicklungen (4. Aufl.). Bad Heilbrunn: Klinkhardt.

Trautmann, M. \& Wischer, B. (2011). Heterogenität als Herausforderung für das Lehrerhandeln im Unterricht. In M. Trautmann \& B. Wischer (Hrsg.), Heterogenität in der Schule. Eine kritische Einführung (S. 105-136). Wiesbaden: Springer VS. doi:10.1007/978-3-531-92893-7

Wocken, H. (2013). Das Haus der inklusiven Schule. Baustellen - Baupläne - Bausteine (4. Aufl.). Hamburg: Feldhaus.

Wylie, E. C., Gullickson, A., Cummings, K., Noakes, L., Egelson, P., Norman, K. et al. (2012). Improving Formative Assessment Practice to Empower Student Learning. Thousend Oaks: Corwin. doi:10.4135/9781452275437

\section{Kontakt}

René Schroeder, Universität zu Köln, Department für Heilpädagogik und Rehabilitation, ClassenKappelmann-Str. 24, 50931 Köln

E-Mail: rene.schroeder@uni-koeln.de

\section{Zitation}

Schroeder, R.; Blumberg, E.; Kottmann, B.; Miller, S. \& Reh, A. (2021). Chancen des inklusionsorientierten Sachunterrichts für didaktisch-diagnostisches Handeln - Konzeptionelle und methodologisch-methodische Grundlagen eines forschungsbasierten Entwicklungsansatzes für die Lehrer*innenildung. QfI - Qualifizierung für Inklusion, 3(2), doi: 10.21248/Qfl.74

Eingereicht: 15. April 2021

Veröffentlicht: 15. Dezember 2021

\section{(cc) $\mathrm{BY}-\mathrm{ND}$}

Dieser Text ist lizenziert unter einer Creative Commons Namensnennung - Keine Bearbeitungen 4.0 International Lizenz. 